\title{
Tetra Primer ARMS PCR Optimization to Detect Single Nucleotide Polymorphism of the KLF14 Gene
}

\author{
Ana Sofia Zabala, Miriam Ester Vasquez Gomez, Micaela Fernanda Álvarez, Susana Siewert \\ Laboratory of Diabetes, Department of Biochemistry and Biological Science, School of Chemistry, Biochemistry and Pharmacy, \\ National University of San Luis, San Luis, Argentina \\ Email: ssiewert@unsl.edu.ar
}

How to cite this paper: Zabala, A.S., Gomez, M.E.V., Álvarez, M.F. and Siewert, S. (2017) Tetra Primer ARMS PCR Optimization to Detect Single Nucleotide Polymorphism of the KLF14 Gene. Open Access Library Journal, 4: e4145.

https://doi.org/10.4236/oalib.1104145

Received: November 13, 2017

Accepted: December 2, 2017

Published: December 5, 2017

Copyright $\odot 2017$ by authors and Open Access Library Inc.

This work is licensed under the Creative Commons Attribution International License (CC BY 4.0).

http://creativecommons.org/licenses/by/4.0/

(c) (i) Open Access

\begin{abstract}
GWAS (genome-wide association studies) have associated Type 2 Diabetes Mellitus with the single nucleotide polymorphism (SNPs) rs972283 (A/G) of KLF14 gene with in a global population. The most common techniques used to analyze SNPs are time consuming, multi-step process and require expensive instruments. Therefore, in order to overcome these problems, we have developed a new, rapid and cost effective T-ARMS PCR assay to genotype rs972283. However, the optimization step can be hardworking and laborious. Hence, we propose to demonstrate and discuss critical steps for its development, in a way to provide useful information. In a first step, we design and validate two specific primer pair for T-ARMS PCR. Later, the amplification conditions were optimized for DNA concentration, annealing temperature, Taq DNA polymerase units and primers concentration. The last one was considered the main interference factor for a correct amplification and appropriate band intensity. Finally, the results obtained by T-ARMS PCR were concordant with sequencing. T-ARMS PCR assay developed in our laboratory for genotyping rs972283 (A/G) of KLF14 gene is time saving and cost-effective compared to the available methods used for SNP studies.
\end{abstract}

\section{Subject Areas \\ Diabetes \& Endocrinology}

\section{Keywords}

rs972283 (A/G), KLF14 Gene, Type 2 Diabetes Mellitus

\section{Introduction}

Single nucleotide polymorphisms (SNP) is a DNA sequence variation occurring 
when a single nucleotide is substituted, deleted, or inserted, affecting how humans develop diseases, respond to pathogens, chemicals, drugs, etc. SNP is the simplest form of polymorphism found in human genome; basically affect more than $1 \%$ of the entire population. The SNP is estimated to occur in every 500 1000 base pairs in the human genome, and may occur both in coding and noncoding region. Although many SNPs showed no effect to cell abnormalities, many researches by statistical calculation found that SNPs can be used as a biological marker for analyzing the disease risks [1] [2].

Gene polymorphism studies have revealed that multiple SNPs are implicated in the development of T2DM. Therefore, genetic risk factors of T2DM need to be explored for T2DM prevention and treatment. KLF14 belongs to the Kruppel-like family of transcription factors. A biological study has demonstrated that KLF14 could participate in the metabolism as a transcriptional activator via regulating the gene networks involved in lipid metabolism [3]. GWAS (genome-wide association studies) have implicated a group of highly-correlated SNPs including rs972283 (A/G) approximately $47 \mathrm{~kb}$ upstream of the transcription-starting codon of the KLF14 gene [4]. The allele G of rs972283 in KLF14 may be a risk factor for metabolic disease and had a nominal association with Type 2 Diabetes Mellitus in a global population [5] [6] [7] but these results are contradictory in Chinese populations where no association between genetic variants of KLF14 rs972283 and T2DM was detected [7] [8].

The SNP detection method was developed since Restriction Fragment Length Polymorphism (RFLP) involves many steps, DNA sequencing needs expensive instruments, and robust SNP genotyping assay depends on consideration of funding [9].

Current techniques for SNP genotyping of medium and low cost are based on the polymerase chain reaction; nevertheless, many of them require post-PCR manipulations, such as radio-isotopes, restriction enzymes, or are required to two PCRs rounds. The use of these reagents not just increase the methodology price, but also the time spent during its execution [10].

In view of the developing countries for which it is very hard and costly to study by PCR and post-PCR manipulation on a large number of samples for analyses. To curb these outcomes, reliable genotyping method which is sufficiently simple, fast and cost effective must be continuously developed [11] [12]. Tetra primer Amplification Refractory Mutation System Polymerase Chain Reaction (T-ARMS PCR) was optimized to find rapid, efficient, economic and low tech laboratories method for detecting SNPs or mutation [13]. The amplification is a multiplex reaction of two set of primers: the forward outer (FO) and reverse outer (RO) primers amplify a specific amplicon of the target gene, irrespective of the allele at SNP position. The forward inner (FI) and reverse inner (RI) primers with $\mathrm{RO}$ and FO primers respectively generate allele-specific amplicons. These amplicons will be of different sizes, hence easily discriminated on an agarose gel as either homozygote or heterozygote. While the two outer pri- 
mers (FO, RO) ensure the gene specificity and PCR efficiency, the inner outer combination (FO/RI, FI/RO) ensures the allele specificity [14]. The reaction of T-ARMS PCR is run in a single tube and in one PCR step, and genotype variation from SNPs of interest could be visualized directly using common agarose gel electrophoresis [10] [15].

T-ARMS PCR assay would be of particular value for laboratories in developing countries, where special and sophisticate instruments are difficult to get. For that reason our study was aimed to optimize that efficient, simple and low cost technique for genotyping rs972283 (A/G) polymorphism of KLF14 gene, as a result this method can be used to genotype large samples of individuals in order to estimate association of this SNP with persons at risk of developing Type 2 Diabetes Mellitus.

\section{Methods}

\subsection{DNA Extraction}

Control genomic DNA for this study was obtained by venous blood draws from healthy adult volunteers; DNA from 50 subjects (25 female and 25 males) was used for the validation of the technique. DNA was extracted using AccuPrep ${ }^{\circ}$ Genomic DNA Extraction Kit (Bioneer) and quantified using Epoch Microplate Spectrophotometer (Biotek) and run in 1\% gel agarose electrophoresis.

\subsection{Primer Design}

Two special set of primers were designed usingweb-based software accessible from the website (http://primer1.soton.ac.uk/public_html/primer1.html) developed by Ye et al. [13]. The specificity was tested using BLAST program (http://www.ncbi.nlm.nih.gov/blast). The primers used for T-ARMS PCR for detection rs972283 are provided in Table 1.

\subsection{Optimization Steps of Tetra Primer ARMS PCR}

The determining factors for the optimization of T-ARMS PCR method were: the correct concentration of the PCR reagent, the ratio of outer and inner primers, as well as the annealing temperature. Those factors were important to increase the PCR product specificity.

Table 1. Primers and conditions used in T-ARMS PCR genotyping.

\begin{tabular}{|c|c|c|c|c|c|}
\hline$S N P$ & Primer sequence (5' to $\left.3^{\prime}\right)$ & Primer conc. & Melting Temp & Annealing Temp. & Expected products \\
\hline & $\begin{array}{l}\text { Forward outer primer } \\
\text { GTCATAGGTCAAACAGCTAGATATTGGGT }\end{array}$ & $0.04 \mu \mathrm{M}$ & $63^{\circ} \mathrm{C}$ & & \\
\hline rs972283 & $\begin{array}{l}\text { Reverse outer primer } \\
\text { TCTACAGGACCAACTCAAATTATGAGGT }\end{array}$ & $0.04 \mu \mathrm{M}$ & $63^{\circ} \mathrm{C}$ & $60^{\circ} \mathrm{C}$ & Common 437 bp \\
\hline \multirow[t]{2}{*}{$(\mathrm{A} / \mathrm{G})$} & $\begin{array}{l}\text { Forward inner primer (G allele) } \\
\text { TCATTGTATACTTGGAAAAAATCCTACATG }\end{array}$ & $0.16 \mu \mathrm{M}$ & $62^{\circ} \mathrm{C}$ & & $\mathrm{G}$ allele $221 \mathrm{bp}$ \\
\hline & $\begin{array}{l}\text { Reverse inner primer (A allele) } \\
\text { TATGTAAAAATAAGTATGCGCCATGCCT }\end{array}$ & $0.04 \mu \mathrm{M}$ & $65^{\circ} \mathrm{C}$ & & A allele 274 bp \\
\hline
\end{tabular}

Underline bases is CG sequence near the 3' end. Bold letter indicate mismatch base. 
Amplification of outer primers region: the reaction was performed in a total volume of $30 \mu \mathrm{l}$, containing $50-100 \mathrm{ng}$ of template DNA, $0.1 \mu \mathrm{M}$ of each outer primer, $200 \mu \mathrm{M}$ dNTPs (Invitrogen, CA, USA), $2.5 \mathrm{mM} \mathrm{MgCl}_{2}, 1 \times \mathrm{PPCR}$ buffer (20 mM Tris- $\mathrm{HCl} \mathrm{pH} \mathrm{8.4,50} \mathrm{mM} \mathrm{KCl}$ ), and $0.7 \mathrm{U}$ of Taq DNA polymerase (Productos Biológicos, Argentina).

$T$-ARMS PCR reaction: It was performed in a total volume of $25 \mu \mathrm{l}$ containing $50-100 \mathrm{ng}$ of template DNA, $0.04 \mu \mathrm{M}$ of Reverse inner primer and both Outer primers, $0.16 \mu \mathrm{M}$ of Forward inner primer, $200 \mu \mathrm{M}$ dNTPs (Invitrogen, CA, USA), $2.5 \mathrm{mM} \mathrm{MgCl} 2,1 \times$ PCR buffer (20 mM Tris- $\mathrm{HCl} \mathrm{pH} \mathrm{8.4,} 50 \mathrm{mM}$ $\mathrm{KCl}$ ), and $0.7 \mathrm{U}$ Taq polymerase (Productos Biológicos, Argentina).

ARMS PCR modified reaction: Allele A amplification: It was performed in a total volume of $25 \mu \mathrm{l}$ containing 50 - $100 \mathrm{ng}$ of template DNA, $0.04 \mu \mathrm{M}$ of Forward outer and Reverse inner primers, $200 \mu \mathrm{M}$ dNTPs (Invitrogen, CA, USA), $2.5 \mathrm{mM} \mathrm{MgCl}$, 1x PCR buffer (20 mM Tris- $\mathrm{HCl} \mathrm{pH} \mathrm{8.4,50} \mathrm{mM} \mathrm{KCl),} \mathrm{and} 0.7 \mathrm{U}$ Taq polymerase (Productos Biológicos, Argentina). Allele $G$ amplification: a total volume of $25 \mu \mathrm{l}$ containing 50 - $100 \mathrm{ng}$ of template DNA, $0.04 \mu \mathrm{M}$ of Reverse outer primer, $0.16 \mu \mathrm{M}$ of Forward Inner primer, $200 \mu \mathrm{M}$ dNTPs (Invitrogen, CA, USA), $2.5 \mathrm{mM} \mathrm{MgCl} 2,1 \times$ PCR buffer (20 mM Tris-HCl pH 8.4, $50 \mathrm{mM}$ $\mathrm{KCl}$ ), and $0.7 \mathrm{U}$ Taq polymerase (Productos Biológicos, Argentina).

Termocycler program: The samples were incubated for $3 \mathrm{~min}$ at $94^{\circ} \mathrm{C}$, followed by 30 cycles of 1 min denaturation $\left(94^{\circ} \mathrm{C}\right), 1 \mathrm{~min}$ annealing $\left(60^{\circ} \mathrm{C}\right)$, and 1 min extension $\left(72^{\circ} \mathrm{C}\right)$, and an additional $10 \mathrm{~min}$ extension at $72^{\circ} \mathrm{C}$ at the end of the 30 cycles.

\subsection{Gel Electrophoresis}

The resultant products obtained after PCR were separated by electrophoresis on 3\% agarose gel, stained with Gel Red (Biotium) against DNA marker 100 - 3000 $\mathrm{pb}$. The images were visualized and photographed under UV transillumination.

\subsection{Validation Assay}

It was done by DNA sequencing of rs972283. For DNA sequencing, conventional PCR was generated to amplify region contain rs972283, using the same Outer primers of T-ARMS PCR (Table 1). The reaction used to amplify the region of outer primers was performed in a total volume of $30 \mu \mathrm{l}$. For genotyping the polymorphism of SNP rs972283, the PCR products were sent to Macrogen Laboratories (Rep. of Korea) for the processing of the samples.

\section{Results and Discussion}

The Amplification Refractory Mutation System (ARMS) is a rapid and reliable method for analysis of point mutations or small deletions. ARMS were originally developed for allele specific amplification avoiding the use of restriction enzymes and radioisotopes [11]. ARMS-PCR enables the identification of specific genotypes in a single step PCR, without the need for costly and often difficult 
post-PCR manipulation.

Ye et al. [16] was the first to describe tetra-primer PCR for which allele-specific amplification is achieved in a single PCR reaction using four primers. This approach introduced a mismatched base in the middle of allele-specific primers. Ye et al. [13] combined tetra-primer PCR with ARMS to form the tetra-primer ARMS-PCR or T-ARMS technique. T-ARMS combines two inner SNP-specific primers and two outer primers in a single reaction and encompasses deliberate mismatches at position -2 from the 3 ' end of inner primersto increase the specificity of the amplification.

T-ARMS PCR primer pairs were designed using Primer 1 software (http://primer1.soton.ac.uk/primer1.html). The inner T-ARMS PCR primer pair was designed with a deliberate mismatch to increase allele specificity [13]. The program follows the Little [17] rules for selecting the additional mismatch base. Under this scheme "strong" mismatches at the 3 ' terminus (A/G or C/T mismatches) are optimally paired with "weak" second mismatches (C/A or G/T) and vice versa with "medium, medium" strength combinations (A/A, C/C, G/G or $\mathrm{T} / \mathrm{T}$ ) as an alternative. $\mathrm{Tm}$ is calculated using the nearest neighbor parameters [18] and the formula given by Rychlik et al. [19].

The Primer1 software operates through web interface input of a target DNA sequence. The required sequence size is of up to 1000 bases which is presented 5' to 3'. The sequence of the target segment was obtained from NCBI Reference Sequence > NC_000007.14, position 130781095 - 130783095. It is located upstream of KLF14 gene and it was used to design primers for the A/G polymorphism (position 534 in this sequence) (Figure 1).

Whitespace and all numerical digits within the sequence were ignored. The sequence location of the polymorphic site is input along with the bases for the reference and the alternative allele. Also inputs have some criteria for primer design: the desired minimum, maximum and average melting temperatures for a primer oligo $(\mathrm{Tm})$; the millimolar concentration of salt $(\mathrm{KCl})$ and the nanomolar concentration of annealing oligos in the PCR (this is used in the calculation of $\mathrm{Tm}$ ); the \%GC content (the minimum and maximum allowable for any primer); the primer lengths and the minimum and maximum complementarity and product sizes. The algorithm used for calculating complementary is similar to Rozen and Skaletsky [20]. The maximum complementarity (default 8.0) is a limit to reduce the possibility of a single primer self-annealing and the possibility of annealing between left and right primers. A total primer-primer annealing score of zero reflects no local alignment between two oligonucleotides. Annealing scores are summed across bases which are scored as: 1.0 for complementary bases; -0.25 for a match with an "N"; -1.0 for a mismatch; -2.0 for a single base gap. The maximum 3' complementarity (for which the default is 3.0 ) follows the same scoring system and measures the maximum allowed 3'-anchored global alignment score [25]. Figure 2 shows the flow chart of the tetra-primer ARMSPCR primer design software [13] and the Input of the sequence location of the polymorphic site (position 534) of KLF14 and the alternative alleles (A/G). 
>NC_000007.14:130781095-130783095 Homo sapiens chromosome 7, GRCh38.p7 Primary Assembly GGAATGTAGGATTGCCCTCCTTACTCATTGCACCCTCCCTGCCCAGAATCTTTAAGT AATAAATATCTGAAGATATTTCCTATTGTGGTAGTGCACTAAATTTGCATCTTACTCT GAAGAACCAGGGGCTCCCCTGGGCCAGGTTCTCTCTGGGATGCTAGAGAAAACAG GGTTGGGTTCCCAGTGCCAGAGCAGTAGTCAAGCAGGCATAAACAGAACACAGGT CAGATATGAGCCACAAGGACCCCTGCTAATATAAACAAGTTTCCCATGTGAGGGAC ACCCTGGTCATAGGTCAAACAGCTAGATATTGGGTTATCTGCCAGGTAAAAGAAGT ATTCTGTGAAAGACACACAGTAAATACCCATGTCAGGCTCCTCCCCATTTCTCATCA GTGCAGGGTCTCTAGCTGCTCTGCTACTGGAATCCCAGTTTAGCTGGAGTCTCTCG GAACAGTCCGTTAATATGGTTGATTGCATTGGTTGATTTTCAGCTATTGAACCATCA TTGTATACTTGGAAAAAATCCTACCT[GIA]GTCATGGCGCATACTTATTTTTACATA TTACTAAACTGTATTTGCTAATATTTTGTTTAAAATTTTGCGTCTGTATTCGTGACAGA TCTTCCTCССТСССТСССТСССТСССТССTTTCСTCTTTGTATTTTCTTGGTCAAGTT TTGATAATAGTGAAACTGACCTCATAATTTGAGTTGGTCCTGTAGAATTAGTGTTAA TGATTCTTTAAACATTTGGTAGGACCCTGTGTCTTCAAATTTGTTGATTCTTTCCACT GTCCTCTCCATTTTGGTATTGAGCCTATATCCATTGAGTTTTCAAATTTTTGGTTATTTT TTTTTCTCTTTCTTTTTTTTTGTTTTTGAGACAGAGTCTTGCTCTGTTGCCCAGGCTG GAGTACAGTGGCAGTCTCAGCTCACTGCAACCTCCACCTCTCGGGTTCAAGCTATT CTCCTGCTTCAGCATCCCAAGTAGCTGGTATTACAGGCATGCGCCACCATGCCTGC AGGCTAAGTTTCGCATTTTTAATAGAGACAGGGTTTCCCCATATTGGCCAG

(a)

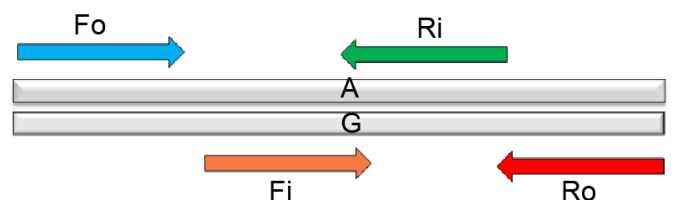

(b)

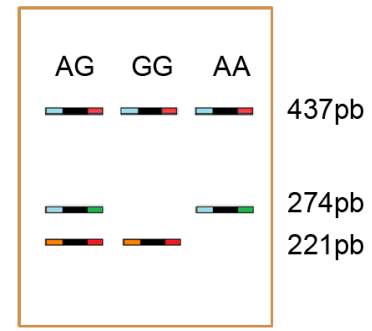

(c)

Figure 1. (a) DNA sequence used to design primers for rs972283 polymorphism. The colored sequence area indicates the position where primers match the sequence. Light blue: Primer Forward Outer (FO), Green: Reverse Inner primer (RI), Orange: Forward inner primer (FI), Red: Reverse Outer primer (RO). (b) Schematic illustration of the tetra-primer T-ARMS PCR assay for SNP genotyping. Primers: Forward outer (FO); Reverse outer (RO), Forward inner (FI) and Reverse Inner ( $R \boldsymbol{I})$. X: deliberate mismatch. (c) Products expected from T-ARMS PCR, discriminated by gel electrophoresis. (Adapted from Ye et al. [13]).

Subject to the user input settings, the program identifies primer sets in sequence of Ye et al. [13]. Firstly, all of the possible inner forward and inner reverse primers are identified. For this set an "optimal” inner primer pair is identified which has the closest match to the input Tm and also the minimum Tm difference between the two primers. With default settings the following primer set was chosen (Table 1). T-ARMS primers were verified for specificity using the Primer-BLAST online application.

The tetra-primer ARMS-PCR method was successfully applied to classify rs972283. The optimal PCR conditions and fragment sizes of the A and G alleles for the KLF14 gene are shown in Table 1.

The allele-specific segments differed sufficiently in size to be distinguished by $3 \%$ agarose gel electrophoresis. For that reason was possible to differentiate between the G and A alleles of the KLF14 gene by means of the T-ARMS PCR 


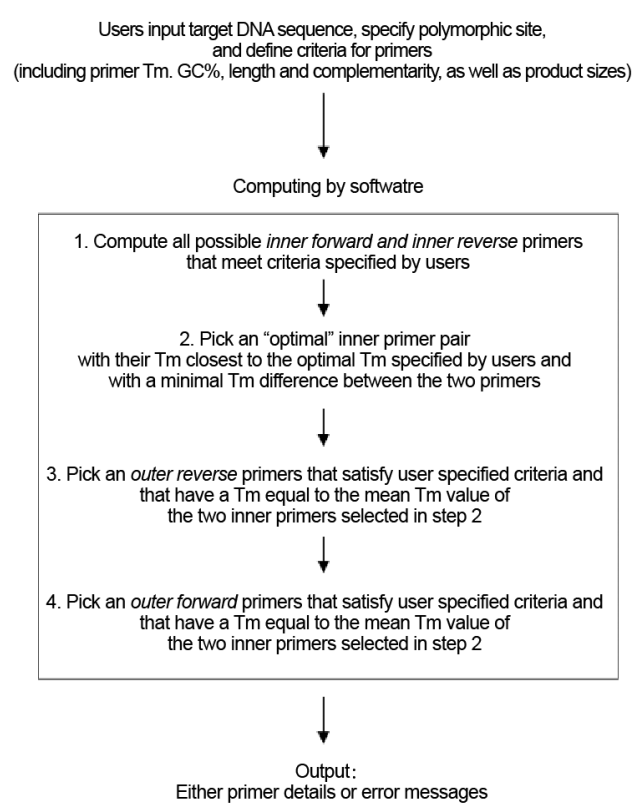

(a)
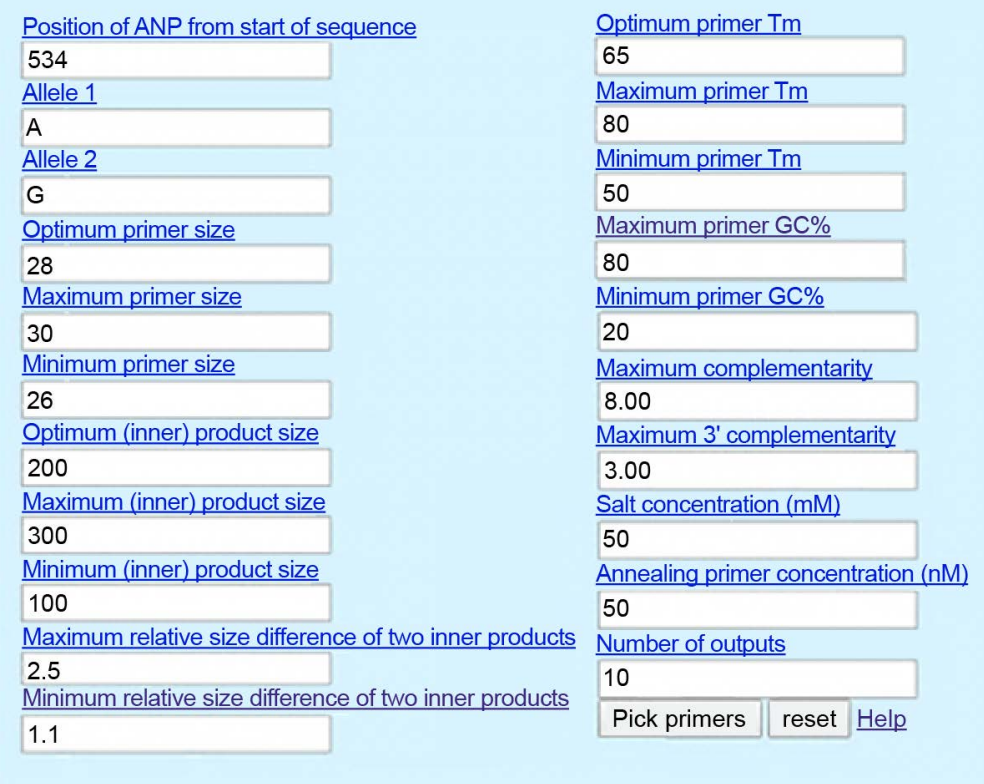

(b)

Figure 2. (a) Flow chart of the T-ARMS PCR primer design program (Ye et al. [13]); (b) Input of the sequence location of the polymorphic site (position 534) and the alternative alleles (A/G).

methodology. The interactions between the inner and outer primers affect both the yield and the specificity of the reaction. Therefore the primer design is a critical step for the success of the T-ARMS PCR method. The inner primers were developed with an average length of 29 bases with a deliberate mismatch at position 2 from the 3'-end. The mismatched bases are shown in bold letter in Table 1.

The development of the T-ARMS PCR was divided into four stages. In the first stage we optimized the right amplification of the outer primers amplicon. This fragment was used as a template for the inner primers, in order to produce the allele specific fragments in T-ARMS PCR. We successfully obtained the fragment of $437 \mathrm{pb}$ (data not shown).

The second stage was to evaluate de optimal composition of each T-ARMS PCR reaction and elaborate protocol changes for genotyping different samples. The objective of this step was to obtain the expected theoretical products by electrophoresis separation: three bands in heterozygous or two bands in homozygous.

In the thirst stage we validated the results by sequencing. Thereby we obtained control samples of known genotype to optimize the experimental condition for a certain analysis of the samples by T-ARMS-PCR.

In the fourth stage we used modified standard ARMS, placing the primers pair for the specific allele amplification separately (FO/RI and FI/RO). Thereby we could design a simple and economical method to validate T-ARMS PCR when the results obtained were uncertain.

One of the key points for the success of the method is that oligonucleotides with a mismatched 3' residue do not function as PCR primers under appropriate 
conditions. Thus, for the allele specific primers, the normal primer is refractory to PCR on mutant DNA and the mutant specific primer is similarly non-functional on normal DNA. Newton et al. [11] noted that, in some cases, having only the single 3 ' mismatched base was insufficient to prevent undesired amplification so they deliberately introduced additional mismatched bases near the 3 ' end.

One of the inconvenients that difficult the genotipification of the samples was the fact that the RI primer (specific for A allele) has GC-rich sequences near the refractory mutation, producing unspecific amplifications of $\mathrm{A}$ allele in all the samples, independently of the genotype. After analyzed 30 samples we observed a characteristic pattern of three types of samples (Figure 3). One of them had three high intense bands that we associated with a possible heterozygous (sample 2 ). The other ones had an intense band that correspond with the A allele size (sample 1 and 3). However sample 1 has a smaller and lower intense band, associated with $\mathrm{G}$ allele (shown with red arrowhead). These results complicated the genotyping of the samples, because we could not identify if it was a heterozygote sample or homozygote with unspecific band.

T-ARMS PCR results require a validation assay to confirm the specificity of the assay. The results obtained by T-ARMS PCR were confirmed with DNA sequencing (Figure 4). Sample 3 shows concordance in genotype obtained in T-ARMS PCR and sequencing, whereas samples 1 and 2 show no coincidence with both methodologies.

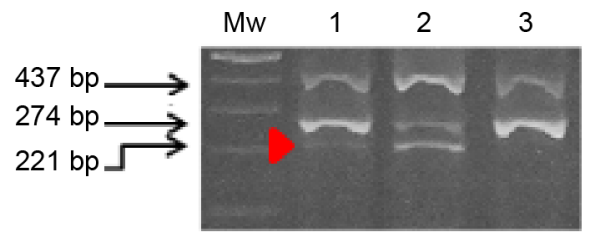

Figure 3. Preliminary genotyping of the SNP rs972283 (A/G) by T-ARMS PCR. Lane 1: Sample 1, Lane 2: Sample 2, Lane 3: Sample 3. Note that Sample 1 has a smaller and lower intense band, associated with $\mathrm{G}$ allele (shown with red arrowhead). Mw: DNA Ladder 100 -3000 bp.

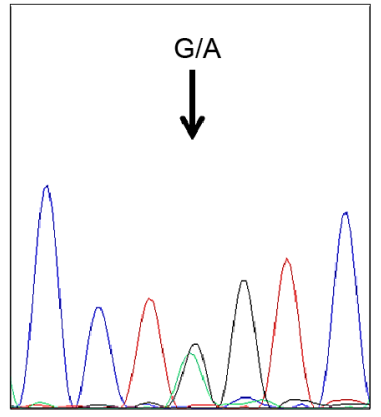

Sample 1

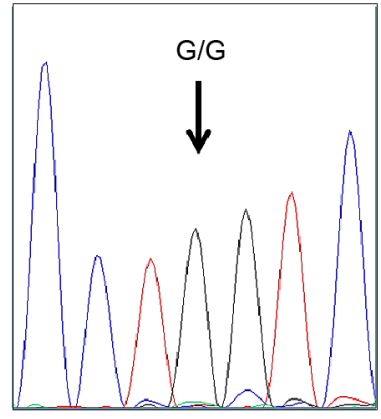

Sample 2

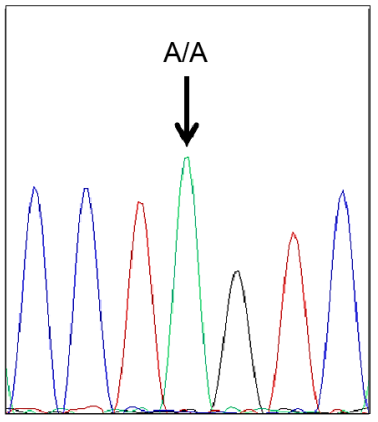

Sample 3

Figure 4. Validation assay to confirm T-ARMS PCR result by using sequencing. Sequencing result of rs972283 showed sample 1 heterozygote $A / G$, homozygote $G / G$ and A/A genotypes, respectively. 
To correct the lack of agreement in the results of samples 1 and 2, different strategies were implemented. We started with the determination of the optimal annealing temperature $(\mathrm{Ta})$.

The melting temperature $(\mathrm{Tm})$ should be considered one of the most important factors in order to achieve an allele-specific amplification [21]. The main difficulty to amplify the correct product of primers with GC-rich sequences was needed high temperatures to break up the strong secondary structure, formed by hydrogen bonds between the cytosine and guanine. During the PCR melting step, the DNA strands begin to separate in a lower Tm, eventually the primers that have a high Tm will not be completely disnatured and the DNA template will be partially open inhibiting the ability of the Taq DNA polymerase to act in those regions [22]. McDowell et al. [23] demonstrated that GC-rich sequences, in conjunct with high $\mathrm{Tm}$, leads to the formation of stable secondary structures in primers, which can reduce the PCR efficiency by serving as termination or stop sites.

The primers used for SNP rs 972283 did not have a high concentration of CG, ranging from $30 \%$ (forward inner primer) to $39 \%$ (outer primers), with a $\mathrm{Tm}$ ranging from $62^{\circ} \mathrm{C}$ to $65^{\circ} \mathrm{C}$.

To identify the optimal annealing temperature we ran 9 PCR reactions with a temperature gradient from $54.6^{\circ} \mathrm{C}$ to $64^{\circ} \mathrm{C}$. The gradient reaction allowed us to observe the amplification of each primer in the set as they approach their optimal Tm. Therefore, to optimize the annealing temperature we preceded by running gradient PCR amplifications with DNA of a heterozygote sample. The gradient PCR experiment allowed a quick determination of the optimal annealing temperature for a given reaction as depicted in Figure 5. When the three expected PCR fragments from a heterozygote were observed in the range of several temperatures; one optimal annealing temperature was chosen. We consider the gradient range $58.8^{\circ} \mathrm{C}-63.5^{\circ} \mathrm{C}$ to be optimal for differentiation of the PCR fragments from a heterozygote, hence the optimal annealing temperature chosen was $60^{\circ} \mathrm{C}$ (Table 1$)$.

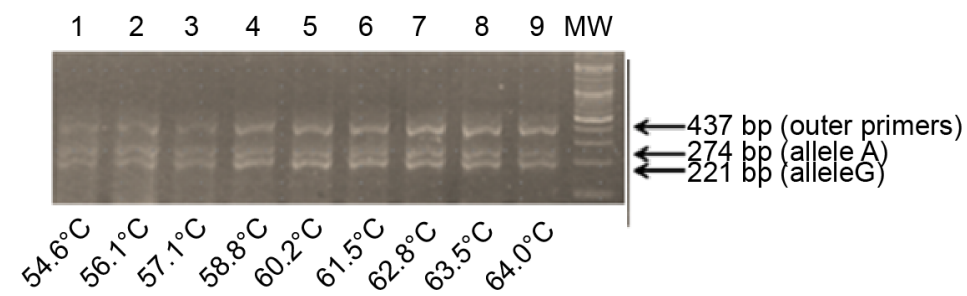

Figure 5. PCR product amplified from a temperature gradient profile utilized for optimizing the KLF14 gene T-ARMS PCR system. The same PCR reagents concentration was used in all samples. A heterozygote individual was amplified with a temperature gradient from $54.6^{\circ} \mathrm{C}$ to $64^{\circ} \mathrm{C}$ (lanes $1-9$ ). Note that in lines $1-3$, the allelic bands are weak. In lanes $4-8$ the two allelic bands (221 and $274 \mathrm{bp}$ ) and common band (437 bp) are present and the profile is perfectly clear. In lane 9 the single lower allele (221 bp) band and common band (437 bp) are present and the $274 \mathrm{bp}$ band is not clear. Mw: DNA Ladder 100 $3000 \mathrm{bp}$. 
Balancing the primer concentration was another important step to obtain specifics and sharp band, for a correct genotyping of the samples. The formation of outer primer fragment reacts strongly to small changes in its concentration and it is critical for the success of the reaction, since the inner primers binds on the outer primer amplicon [13].

Proceeding with the $60^{\circ} \mathrm{C}$ Ta, the outer primers fragment was stabilized and the intensity of the two inner primer amplicons balanced. For that reason, a pattern among the intensity of the fragments and the each primers concentration could be designed. Optimization of T-ARMS PCR was applied by using different ratios of outer and inner primer concentration in $1: 1(0.75 \mu \mathrm{M}), 1: 2(0.08 \mu \mathrm{M}$ and $0.16 \mu \mathrm{M}), 1: 5(0.08 \mu \mathrm{M}$ and $0.43 \mu \mathrm{M})$ and $1: 10(0.08 \mu \mathrm{M}$ and $0.85 \mu \mathrm{M})$. In our assay, high concentrations of outer primers inhibited the formation of the expected fragment of $437 \mathrm{bp}$ (data not shown). Changes in outer primers concentration were sufficient to modify the fragment amplification and their intensity. A $0.08 \mu \mathrm{M}$ concentration of outer primers allowed the obtaining of the 437 bp fragment, however the smaller fragment of $221 \mathrm{bp}$ ( $\mathrm{G}$ allele) did not have good intensity of the band.

The final primer concentration for rs972283 detection was triplicate the Forward inner concentration respect the others primers (Table 1).

The mutant and wild type allele were distinguished by the fragment size of T-ARMS PCR product. The size of DNA fragments produced by a pair of common outer primers (non-allele-specific control band) and their combination with 2 allele-specific inner primers (the wild-type and the mutant amplicons) in simultaneous amplification can be separated by standard gel electrophoresis. It is possible because of allele-specific inner primers were designed to anneal in the opposite orientation and they are asymmetrically located with respect to the common primers. The product of T-ARMS PCR specific for rs 972283 contained $437 \mathrm{bp}$ as a control fragment, $274 \mathrm{bp}$ as A allele (mutant), and $221 \mathrm{bp}$ as G allele (wild type). In this report, we suggest that the DNA fragments between two specific allele could clearly separate if they have minimum 50 bp (Figure 6).

Garce's-Claver and collaborators [24] reported that the smaller fragment of the inner primers is disadvantaged by the formation of larger fragments, resulting in a weaker intensity of the band. In our case, with the same concentrations of inner primers, not only the intensities of the allelic bands were different (the

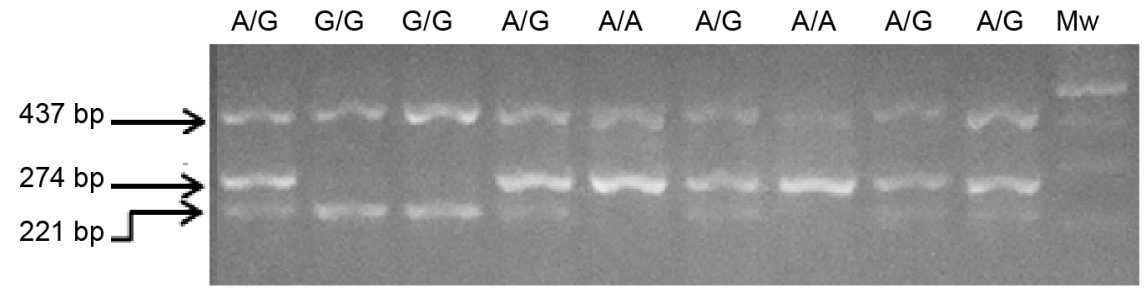

Figure 6. T-ARMS PCR for rs972283. Genotype variation determined by control fragment (437 bp), specific fragment of A allele (274 bp) and G allele (221 bp); and Mw: DNA Ladder 100 - 3000 bp. 


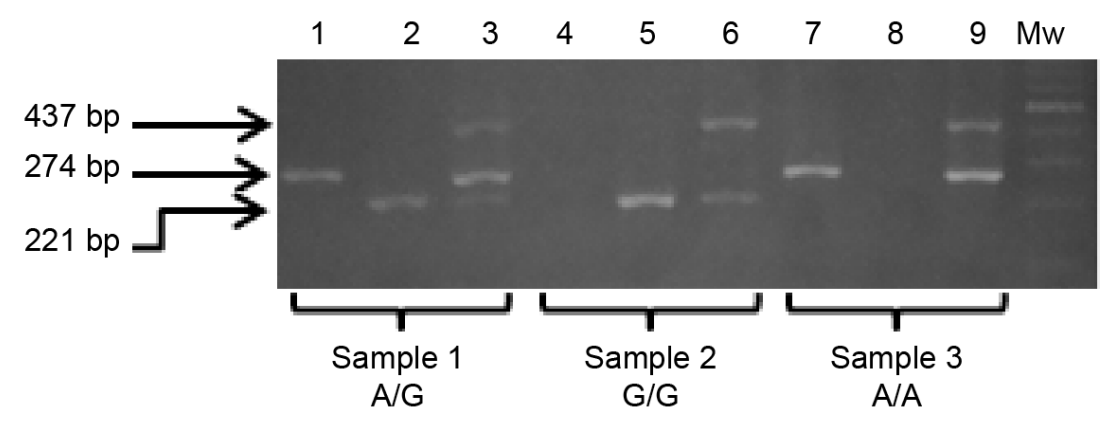

Figure 7. Validation assay to confirm T-ARMS PCR result by modified standard ARMS PCR. Lanes 1, 4 and 7(A allele amplification); lanes 2, 5 and 8 (G allele amplification); lanes 3, 6 and 9 (T-ARMS PCR); and Mw: DNA Ladder $100-3000$ bp.

A allele showed higher intensity respect to the $G$ allele) but also the amplification of both alleles was observed in the homozygous G/G by ARMS modified PCR (data not shown). The analysis of reverse inner primer allowed us to observe that it has a CG sequence near the 3 'end, which would probably allow the unspecific binding with DNA, in spite of the deliberate mismatch (underlined in Table 1).

A decrease in concentration of the reverse inner primer (A allele) versus concentration of forward inner primer ( $G$ allele) allowed correct identification of genotype using as a control the sequencing results. We apply the methodology in the analysis in more of 30 samples and the results allow the easy identification of different genotypes with a simple PCR reaction and electrophoresis separation (Figure 6). The reproducibility of the method was determined in five independent PCR reactions on three different days. For the tested gene, wild-type, heterozygous and homozygous positive controls were used. We observed complete concordance between each test.

Finally, T-ARMS PCR for rs972283 detection it was also confirmed by modified standard ARMS PCR. Full accordance was found between both methods for genotype determination (Figure 7).

Randomly selected $20 \%$ of samples were re-genotyped for cross validating initial genotypes by T-ARMS PCR and modified standard ARMS PCR. Whenever, genotyping results were unclear, the samples were repeated again in duplicates until clear genotype was obtained. No genotyping error was observed during cross validation.

\section{Conclusions}

In this study, T-ARMS PCR protocol was successively and thoroughly optimized in terms of PCR cycling conditions and reaction components for SNP genotyping, with minimally equipped laboratories. We conclude that T-ARMS PCR is a robust, simple and relatively economic method for genotyping of rs972283. Furthermore T-ARMS PCR is useful for large scale of SNP-based association study in different population to understand the genetics risk factor of Type 2 Diabetes Mellitus and metabolic disease. 
The critical steps to improve the specific amplification of the allelic variant of the SNP were primer design, the ratio of outer and inner primers and their concentration, and the annealing temperature used in PCR reaction.

\section{Acknowledgements}

This research was supported by grants from the National University of San Luis (SES-PROICO 2-2814).

\section{Conflict of Interests}

The authors declare no conflict of interests with respect to the present paper.

\section{References}

[1] Fareed, M. and Afzal, M. (2012) Single Nucleotide Polymorphism in Genome-Wide Association of Human Population: A Tool for Broad Spectrum Service. Egyptian Journal of Medical Human Genetics, 14, 123-134. https://doi.org/10.1016/j.ejmhg.2012.08.001

[2] Iversen, E.S., Lipton, G., Clyde, M.A. and Monteiro, A.N. (2014) Functional Annotation Signatures of Disease Susceptibility Loci Improve SNP Association Analysis. BMC Genomics, 15, 398. https://doi.org/10.1186/1471-2164-15-398

[3] de Assuncao, T.M., Lomberk, G., Cao, S., Yaqoob, U., Mathison, A., Simonetto, D.A., et al. (2014) New Role for Kruppel-Like Factor 14 as a Transcriptional Activator Involved in the Generation of Signaling Lipids. The Journal of Biological Chemistry, 289, 15798-15809. https://doi.org/10.1074/jbc.M113.544346

[4] Voight, B.F., Scott, L.J., Steinthorsdottir, V., Morris, A.P., Dina, C., Welch, R.P., et al. (2010) Twelve Type 2 Diabetes Susceptibility Loci Identified through Large-Scale Association Analysis. Nature Genetics, 42, 579-589. https://doi.org/10.1038/ng.609

[5] Ohshige, T., Iwata, M., Omori, S., Tanaka, Y., Hirose, H., Kaku, K., Maegawa, H., Watada, H., Kashiwagi, A., Kawamori, R., Tobe, K., Kadowaki, T., Nakamura, Y. and Maeda, S. (2011) Association of New Loci Identified in European Genome-Wide Association Studies with Susceptibility to Type 2 Diabetes in the Japanese. PLoS One, 6, e26911. https://doi.org/10.1371/journal.pone.0026911

[6] Small, K.S., Hedman, A.K., Grundberg, E., Nica, A.C., Thorleifsson, G., Kong, A., et al. (2011) Identification of an Imprinted Master Trans Regulator at the KLF14 Locus Related to Multiple Metabolic Phenotypes. Nature Genetics, 43, 561-564. https://doi.org/10.1038/ng.833

[7] Wang, J., Zhang, J., Shen, J., Hu, D., Yan, G., Liu, X., Xu, X., Pei, L., Li, Y. and Sun, C. (2014) Association of KCNQ1 and KLF14 Polymorphisms and Risk of Type 2 Diabetes Mellitus: A Global Meta-Analysis. Human Immunology, 75, 342-347. https://doi.org/10.1016/j.humimm.2014.01.008

[8] Gao, K., Wang, J., Li, L., Zhai, Y., Ren, Y., You, H., Wang, B., Wu, X., Li, J., Liu, Z., Li, X., Huang, Y., Luo, X.P., Hu, D., Ohno, K. and Wang, C. (2016) Polymorphisms in Four Genes (KCNQ1 rs151290, KLF14 rs972283, GCKR rs780094 and MTNR1B rs10830963) and Their Correlation with Type 2 Diabetes Mellitus in Han Chinese in Henan Province, China. International Journal of Environmental Research and Public Health, 26.

[9] Kwok, P.Y. and Chen, X. (2003) Detection of Single Nucleotide Polymorphisms. Current Issues in Molecular Biology, 5, 43-60. 
[10] Medrano, R.F.V. and de Oliveira, C.A. (2014) Guidlines for the Tetra Primer ARMS-PCR Technique Development. Molecular Biotechnology, 56, 599-608.

[11] Newton, C.R., Graham, A., Heptinstall, L.E., Powell, S.J., Summers, C. and Kalsheker, N. (1989) Analysis of Any Point Mutation in DNA: The Amplification Refractory Mutation System (ARMS). Nucleic Acids Research, 17, 2503-2516. https://doi.org/10.1093/nar/17.7.2503

[12] Okayama, N., Fujimura, K., Nakamura, J., Suehiro, Y., Hamanaka, Y. and Hinoda, Y. (2004) Evaluation of a New Efficient Procedure for Single-Nucleotide Polymorphism Genotyping: Tetra-Primer Amplification Refractory Mutation System-Polymerase Chain Reaction. Clinical Chemistry and Laboratory Medicine, 42, 13-16. https://doi.org/10.1515/CCLM.2004.004

[13] Ye, S., Dhillon, S., Ke, X., Collins, A.R. and Day, I.N. (2001) An Efficient Procedure for Genotyping Single Nucleotide Polymorphisms. Nucleic Acids Research, 29, E88-8. https://doi.org/10.1093/nar/29.17.e88

[14] Alyethodi, R.R., Singh, U., Kumar, S., Deb, R., Alex, R., Sharma, S., Sengar, G.S. and Prakash, B. (2016) Development of a Fast and Economical Genotyping Protocol for Bovine Leukocyte Adhesion Deficiency (BLAD) in Cattle. Springerplus, 5, 1442. https://doi.org/10.1186/s40064-016-3148-7

[15] Akhlawat, S., Sharma, R., Maitra, A., et al. (2014) Designing, Optimization, and Validation of Tetra Primer ARMS PCR Protocol for Genotyping Mutation in Caprine Fc Genes. Metagene, 2, 439-449. https://doi.org/10.1016/j.mgene.2014.05.004

[16] Ye, S., Humphries, S. and Green, F. (1992) Allele Specific Amplification by Tetra-Primer PCR. Nucleic Acids Research, 20, 1152. https://doi.org/10.1093/nar/20.5.1152

[17] Little, S. (1997) ARMS Analysis of Point Mutations. In: Taylor, G.R., Ed., Laboratory Methods for the Detection of Mutations and Polymorphisms in DNA, CRC Press, Boca Raton, 45-51.

[18] Breslauer, K.J., Frank, R., Blöcker, H. and Marky, L.A. (1986) Predicting DNA Duplex Stability from the Base Sequence. Proceedings of the National Academy of Sciences of the USA, 83, 3746-3750. https://doi.org/10.1073/pnas.83.11.3746

[19] Rychlik, W., Spencer, W.J. and Rhoads, R.E. (1990) Optimization of the Annealing Temperature for DNA Amplification in Vitro. Nucleic Acids Research, 18, 6409-6412. https://doi.org/10.1093/nar/18.21.6409

[20] Rozen, S. and Skaletsky, H. (2000) Primer3 on the WWW for General Users and for Biologist Programmers. Methods in Molecular Biology, 132, 365-386.

[21] Chiapparino, E., Lee, D. and Donini, P. (2004) Genotyping Single Nucleotide Polymorphisms in Barley Tetra-Primer ARMS-PCR. Genome, 47, 414-420. https://doi.org/10.1139/g03-130

[22] Hube, F., Reverdiau, P., Iochmann, S. and Gruel, Y. (2005) Improved PCR Method for Amplification of GC-Rich DNA Sequences. Molecular Biotechnology, 31, 81-84. https://doi.org/10.1385/MB:31:1:081

[23] McDowell, D.C., Burns, N.A. and Parkes, H.C. (1998) Localized Sequence Regions Possessing High Melting Temperatures Prevent the Amplification of a DNA Mimic in Competitive PCR. Nucleic Acids Resarch, 26, 3340-3347. https://doi.org/10.1093/nar/26.14.3340

[24] Garce's-Claver, A., Fellman, S.M., Gil-Ortega, R., Jahn, M. and Arnedo-Andre's, M.S. (2007) Identification, Validation and Survey of a Single Nucleotide Polymorphism (SNP) Associated with Pungency in Capsicum spp. TAG Theoretical and 
Applied Genetics, 115, 907-916. https://doi.org/10.1007/s00122-007-0617-y

[25] Andrew, C. and Xiayi, K. (2012) Primer1: Primer Design Web Service for TetraPrimer ARMS-PCR. The Open Bioinformatics Journal, 6, 55-58.

https://doi.org/10.2174/1875036201206010055 